\title{
The effects of divided attention at encoding on item and associative memory
}

\author{
MOSHE NAVEH-BENJAMIN \\ University of Missouri, Columbia, Missouri \\ and Ben-Gurion University of the Negev, Beer-Sheva, Israel \\ and \\ JONATHAN GUEZ and MICHAL MAROM \\ Ben-Gurion University of the Negev, Beer-Sheva, Israel
}

\begin{abstract}
Divided attention at encoding is well known to have adverse effects on episodic memory performance (e.g., Naveh-Benjamin \& Greg, 2000). This article attempts to determine whether these effects are a result of the interruption of encoding of associative information among the components of an episode. Five experiments, using different types of episodes and episodes components, were conducted. Participants studied information under either full or divided attention and were then tested on their memory for both the episodes' components and the associations between them. Divided attention did not produce a differential deficit in memory for associative information; memory for the components suffered to the same degree as memory for the associations among the components. The cause of the dividedattention effect at encoding lies somewhere other than in the associative processes that are engaged.
\end{abstract}

Divided attention (DA) at encoding has been shown to have marked effects on memory performance. For example, dividing participants' attention between the encoding of the information presented and performing a secondary task had a clear detrimental effect on free recall, cuedrecall, and recognition memory performance relative to conditions where full attention (FA) was paid to encoding the items (e.g., Baddeley, Lewis, Eldridge, \& Thomson, 1984; Craik, Govoni, Naveh-Benjamin, \& Anderson, 1996; Murdock, 1965; Naveh-Benjamin, Craik, Guez, \& Dori, 1998; Naveh-Benjamin, Craik, Gavrilescu, \& Anderson, 2000; Naveh-Benjamin, Craik, Perretta, \& Tonev, 2000). DA at encoding has been shown to have a similar effect on a variety of memory features, including memory for frequency of occurrence (Naveh-Benjamin \& Jonides, 1986), memory for spatial location (Naveh-Benjamin, 1987, 1988), and memory for temporal order information (NavehBenjamin, 1990). Moreover, changes in task emphasis result in systematic and complementary changes in performance in both tasks (Craik et al., 1996; Murdock, 1965).

This research was supported in part by a grant from the Ben-Gurion University Faculty of Humanities and Social Sciences as well as a grant from the Zlotowski Center for the Neurosciences. We are grateful to members of the memory research group at Ben-Gurion University of the Negev for their help in conducting the research, and to Fergus Craik, William Wallace, Sachiko Kinoshita, and Riccardo Russo for their helpful comments on an earlier version of this manuscript. This manuscript was written while M.N.-B. was a visiting scientist at the Rotman Research Institute, Baycrest Centre for Geriatric Care, Toronto, Canada. Correspondence should be addressed to M. Naveh-Benjamin, Department of Psychological Sciences, University of Missouri, Columbia, MO 65211 (e-mail: navehbenjaminm@missouri.edu).
These results indicate that the encoding process requires attention and that the allocation of attention to the encoding process is under the individual's conscious control.

Several possible mediator mechanisms have been suggested for the effects of DA at encoding (see NavehBenjamin, 2002, for a review). One, the reduced processing time hypothesis (e.g., Craik et al., 1996), claims that memory performance under DA at encoding decreases because participants are spending some of their time performing the secondary task, leaving less time for processing the relevant information. However, using a shared time model analysis, Craik et al. (1996) showed that the decrease in processing time could explain only part of the memory deficit resulting from DA effects at encoding. Using a similar approach, Naveh-Benjamin, Craik, Gavrilescu, and Anderson (2000) also showed that the decline in memory performance as a result of DA at encoding was only partially related to the reduced functional time available for encoding.

Another possible mediator in the effects of DA at encoding on later memory performance is the level of processing employed by the participants. It makes sense that DA at encoding qualitatively causes encoding to become shallower and less semantically elaborative. This notion was supported behaviorally in a series of studies during the 1980s (e.g., Craik, 1982; Craik \& Byrd, 1982; Rabinowitz, Craik, \& Ackerman, 1982). These studies indicated that DA at encoding changes the qualitative nature of encoding so that it becomes less semantic. More recently, neuroimaging studies (e.g., N. D. Anderson et al., 2000; Fletcher et al., 1995) have shown that DA at encoding reduces encoding-related brain activity in the left inferior pre- 
frontal cortex, an area shown in other studies to be associated with deep strategic semantic processing (e.g., Kapur et al., 1994).

However, several recent studies complicate this picture. For example, Craik and Kester (2000) employed a cued recall paradigm where participants rated the elaboration of the connection that they created for each word pair during study. Results indicated that, in contrast to what is predicted by the elaboration of processing hypothesis, for the same degree of strategic semantic elaboration, fewer words were recalled after being encoded under DA conditions. This implies that there is a mechanism other than the degree of strategic elaboration underlying the effects of DA at encoding on later memory performance. In addition, NavehBenjamin (2002) showed that the effects of DA at encoding were similar (1) when learning was incidental and no deep level strategic processing was used and (2) when learning was intentional and deep elaborative strategies were used. Such results indicate that DA most likely also affects types of processing other than deep effortful ones.

These results leave open the mechanism underlying the effects of DA at encoding on later episodic memory performance. The purpose of the present research was to test another hypothesis regarding the effects of DA at encoding on memory - the associative deficit hypothesis (NavehBenjamin, 2000) - which attributes the detrimental effects of DA at encoding to a disruption of the mechanism responsible for associating different components of an episode into a coherent unit. This hypothesis is based on the notion that complex events consist of multiple kinds of information sources that are related (e.g., Underwood, 1969). The use of task analysis suggests that what is disrupted during DA at encoding is the cohesiveness of the episode created; that is, because participants have to alternate between processing the primary encoding task and the secondary task, they are not capable of uninterrupted encoding and of binding together the different components of the episode. This results in the creation of a fragmented encoding unit. If this is the case, we would expect DA at encoding to prove more disruptive to the associative mechanism, which binds together the components of the episode, than to the mechanism that encodes each component separately.

The literature quite strongly supports a separation within memory of information about single units (items or components) from information about associative relations among these units (e.g., J. R. Anderson \& Bower, 1973; Chalfonte \& Johnson, 1996; Gillund \& Shiffrin; 1984; Humphreys, 1976; Johnson, 1992; Johnson \& Chalfonte, 1994; Murdock, 1982, 1993). This distinction between item and associative information has been shown by several experiments that yielded different patterns of results for the two types of information. For example, Dosher (1988) and Gronlund and Ratcliff (1989) have shown that retrieval of item and associative information has different time courses. In addition, Hockley (1994) has shown that language word frequency has a differential effect on item versus associative information. Also, Hockley $(1991,1992)$ demonstrated that item and associative information show different forgetting rates. Finally, Hockley and Christi (1996) showed that learning instructions have differential effects on item and associative information.

If we want to directly compare memory for item and associative information, we need to choose two tasks that require those processes, with only one difference: Whereas one task must require the encoding and retrieval of associative information, the other must require the encoding and retrieval of item information. In addition, to attribute the difference between item and associative information mostly to encoding, tasks must be chosen that minimize retrieval effects. One such procedure was used by Humphreys (1976; see also Hockley, 1992, 1994; Hockley \& Christi, 1996). Under this procedure, participants study a list of pairs of items. For item information, participants receive at test some of the original items along with some new items, and their task is to recognize the old items. For associative information, participants receive some intact pairs, which appear as pairs at the study phase, and some recombined pairs, composed of items that were presented earlier but now the A item is taken from one pair and the B item from a different pair. Participants have to recognize which of the pairs appeared as such, originally. Participants thus encode the same information at study, and are provided with all of the information in both tests. Hence, the differences in performance between the two tests reflect differential memory for item and associative information. Note that although the tests are probably not process pure (e.g., memory for the components could create familiarity-based false responses for recombined pairs in the associative recognition test), they seem suitable for a comparison of the two types of processing. The two tests do not seem to differ in difficulty, either (see the results of Experiment 2, showing similar secondary task costs for both).

In summary, our aim was to evaluate an associative deficit hypothesis as the locus of the effects of DA at encoding on memory. To this end, we conducted five experiments that examined the effects of DA at encoding on item and associative information using several types of associations, different instructions, and different memory tasks.

\section{GENERAL METHOD}

To increase the external validity of the experiments, we have used a variety of associations. In the first two experiments, we used the episodic relations established between two unrelated items that appeared together (inter-unit associations; Mandler, 1979). Experiment 1 used word-nonword pairs, whereas Experiment 2 used wordword pairs. In Experiment 3, we used the episodic relations established between different memory attributes (memory for word-font pairs; intra-unit associations; Mandler, 1979). In Experiments 4 and 5, we contrasted predictions made by an associative deficit hypothesis to those made by a hypothesis that posits a general poorer encoding under DA. This was done in Experiment 4 by assessing the effects of DA at encoding on different memory tasks, and in Experiment 5 by providing preexperimental semantic support for the creation of associations. To increase the external validity of the experiments and to ensure that the results are not due to a particular type of design, we used different types of designs. This was accomplished by manipulating attention between subjects in Experiments 1 and 3, and within subjects in Experiments 2, 4, and 5.

In all of the experiments, we employed a dual-task paradigm with the following features. First, we used well-understood memory par- 
adigms in which encoding and retrieval phases could be clearly separated. We presented the memory lists either auditorily or visually, depending on the experiment. To avoid modality-specific interference, when the memory lists were presented auditorily, the concurrent task used visual stimuli and manual responses. When the memory lists were presented visually, the concurrent task used auditory stimuli and manual responses. The concurrent task was either a visual or an auditory continuous choice reaction time (CRT) task reported in previous studies (Craik et al., 1996; Naveh-Benjamin et al., 1998; Naveh-Benjamin, Craik, Gavrilescu, \& Anderson, 2000), in which the next stimulus appeared immediately after the participant's response. Such a continuous task does not leave indefinite amounts of time to switch focus to the memory task, unlike other secondary tasks (e.g., detection of occasional targets or card sorting). The fact that performance did not reach ceiling on either task performed singly indicates that each task required full attention to perform on its own. When performed together, the tasks allowed for assessment of performance throughout the dual-task interval. Participants were told in all the experiments to pay equal attention to learning the relevant materials and performing on the secondary task.

Before the experimental trials began, participants in each of the experiments served in a short study phase under FA conditions accompanied by the relevant tests. In addition, they practiced the secondary CRT task alone, as well as a combination of the memory and the CRT tasks at encoding (DA at encoding). Any questions that the participants may have had were answered before they began the experimental trials. At the end of the study phase for each experiment, participants had to count backward by threes for $90 \mathrm{sec}$ as an interpolated activity. In all of the experiments reported in this article, participants were told to provide a response for each test item once they reached a decision. They had as much time as they needed to make their responses. A response triggered the appearance of the next test item.

\section{Experiment 1}

In the first experiment, we assessed the degree to which DA at encoding differentially affects memory for associative and item information. The type of association used in this experiment was the episodic relations established between two unrelated items that appeared together (interunit associations). Unrelated word-nonword pairs were presented for study under either full or divided attention at encoding and under instructions to learn for upcoming memory tests. Item memory was tested by a recognition test in which half of the items (either the words or the nonwords) were studied (targets) and half were not (distractors). Associative memory was tested by presenting participants with target items only, either as intact pairs (a word and a nonword that were presented together at study), or as recombined pairs (a word and a nonword presented in different pairs at study), and asking them to recognize the intact pairs. An associative deficit hypothesis predicts an interaction between attention condition and type of test. Specifically, the differences between FA and DA conditions at encoding are expected to be the greatest in the word-nonword association test.

\section{Method}

Participants. Participants were 60 adult undergraduate students at Ben-Gurion University who participated in the experiment as part of their course requirements. Half were assigned to the FA condition and the other half to the DA condition.

Design. Two independent variables were used: attention (FA vs. DA at encoding; between subjects), and test (words, nonwords, words + nonwords; within subjects).
Materials. In the study phase, a list of 40 word-nonword pairs was presented on the computer screen. Half of the participants learned the list under FA and half under DA. Each word pair was presented at the same vertical level with five horizontal spaces between the word and the nonword. The first 6 pairs served as practice. Of the next 34 pairs, 4 ( 2 at the beginning and 2 at the end) were used as buffers. The remaining 30 pairs served as the experimental stimuli. Words were high-frequency two- and three-syllable Hebrew nouns taken from Balgur's (1968) norms. Each nonword was created by swapping letters of a word not included in the word sample. The resulting nonwords were all pronounceable and similar in length to the words. The word and the nonword in each pair were acoustically and structurally unrelated to each other. Words and nonwords belonging to different pairs were also unrelated to each other in any apparent way. Two random versions of word-nonword pairings were created and two random orders were used for each of these pairings to create four versions. Seven or 8 participants in each of the attention conditions were run in each version. The distractor items in the word and nonword tests were 10 words or nonwords, respectively, with the same characteristics as the targets.

The secondary task was a continuous three-CRT task that involved a sequential presentation of auditory tones by the computer, one at a time, and a manual response on a computer keyboard to each tone. One of three tones, which differed from each other in frequency, was presented at random and the participants' task was to press a predesignated corresponding key on the keyboard. A response triggered the immediate presentation of one of the other two tones at random.

Procedure. Participants, who were tested individually, saw a list of word-nonword pairs presented sequentially. They were told to study them in preparation for the upcoming component (word and nonword) and associative recognition tests, the nature of which was explained.

For each attention condition, the word-nonword pairs were presented sequentially at a pace of one pair every $7 \mathrm{sec}$. After presentation of the first six pairs, which were used for practice, three short tests were given. In the word recognition test, two target words (one from each of two pairs) and two distractors (new words) were given. Likewise, in the nonword recognition test, two nonword targets (one from each of two pairs) and two distractors (new nonwords) were given. The pair recognition test included two intact pairs and two recombined pairs. Participants in the DA condition first performed the secondary auditory task for $90 \mathrm{sec}$ and then were given the practice phase under DA at encoding conditions.

During the next phase, participants were presented with the 34 experimental pairs, one at a time without pause. Participants under the FA condition learned the word pairs alone, whereas those under the DA condition learned them while performing the secondary task. In addition, participants under DA conditions also performed the secondary CRT task alone, half of them before the memory trial and half after it.

After the end of the study phase and the interpolated activity, the three memory tests listed below-one for words, one for nonwords, and one for their associations- were administered to all participants. The order of the tests was counterbalanced across all participants, resulting in 5 participants in each attention condition serving in each of the six orders. Each word, nonword, or word-nonword pair appeared in only one of the tests. This was done to avoid across-tests item reexposure effects.

In the first test (word recognition), participants saw 20 words, one at a time. Of these, 10 were targets and 10 were distractors that were mixed randomly. For each participant, targets were selected at random from 10 of the studied pairs, one from each pair. The distractors were 10 words with the same characteristics as the target words, except that they had not appeared in the study phase. Participants were told that 10 of the words had appeared in the study phase and were instructed to respond to these words with a designated "yes" response key and to the distractor words with a designated "no" key.

The second test, the nonword recognition test, was similar to the word recognition test except that participants saw 20 nonwords, one 
at a time. Of these, 10 were targets and 10 were distractors that were mixed randomly. For each participant, targets were selected at random from 10 of the studied pairs, one from each pair. The distractors were 10 nonwords with the same characteristics as the target words except that they did not appear in the study phase. Participants were told that 10 of the nonwords had appeared in the study phase; they were instructed to respond to these nonwords with a designated "yes" response key, and to the distractor nonwords with a designated "no" key.

Finally, in the third test (associative recognition one), 20 wordnonword pairs, selected randomly for each participant, were presented visually one at a time. Ten of them were intact pairs from the study phase - that is, pairs that appeared together in the study phase. The other 10 pairs were recombined (rearranged) pairs; that is, they were composed of words and nonwords taken from different study pairs. None of these words or nonwords had appeared in the item recognition tests. Participants were told that all items had appeared in the study phase and that their task was to respond "yes" to the 10 pairs that had appeared together and "no" to the 10 pairs that had not appeared together during study.

\section{Results}

Memory performance. Because some of the participants in both attention groups did not provide the suggested number of words as targets (they provided too many or too few), measures of proportion of hits minus false alarms were computed for each participant in this and all other experiments reported here and then averaged over each attention group for every test. This equated the word, nonword, and associative recognition tests with respect to the scale used (from chance level performance at 0 to highest possible score at 1.0). In addition, the three tests employed were of comparable discriminating power, as indicated by the similar variability of performance in the three, allow- ing their direct comparison as three levels of the test variable (for this and the following experiments). Also, because a preliminary analysis of variance (ANOVA) in each of the experiments reported here indicated no interaction effect of order of the administration of the tests with any of the independent variables, in all of the following reported analyses (for this and the following experiments), performance was collapsed across the different orders. Finally, the .05 level of significance was used to interpret all statistical comparisons.

Figure 1 presents results for the proportion of the hits minus false alarm measure (with standard errors) in the different conditions. A 2 (attention) $\times 3$ (test) mixed factorial ANOVA was performed on the corrected hit rates. The effect of attention was significant $[F(1,58)=5.05$, $\left.M S_{\mathrm{e}}=.18\right]$, where participants under FA (.46) performed better than those under DA (.32) at encoding. The effect of test was not significant $\left[F(2,116)=0.74, M S_{\mathrm{e}}=.03\right]$, reflecting the fact that performance did not differ across the word test (.39), the nonword test (.41), and the wordnonword association test (.37). Most importantly for the purpose of the present study, the interaction of attention and test was not significant $\left[F(2,116)=0.22, M S_{\mathrm{e}}=.03\right]$. As can be seen in Figure 1, this absence of interaction reflects similarly reduced memory performance in all three tests under DA conditions. In particular, performance in the word-nonword association test was not affected more by DA at encoding than was performance in either component test. Finally, performance in the DA condition in all tests was significantlybetter than chance level (0), demonstrating that the absence of an interaction was not due to floor effects.

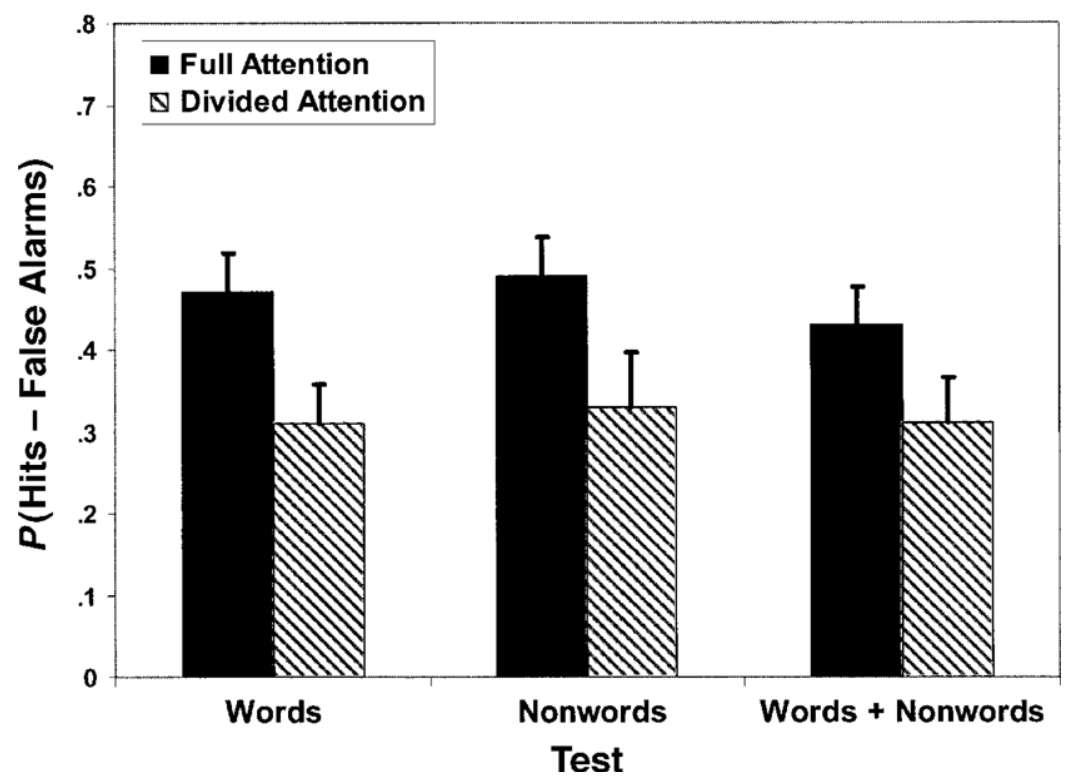

Figure 1. Experiment 1: Proportion hits minus proportion false alarms $(+S E s)$ in the item (word, nonword) and the associative (word + nonword) recognition tests for each attention condition. 
Given that baseline performance of the young adults under FA and DA conditions differed significantly (with the FA group performing at a higher level), it is possible that measures of absolute scores may not reveal the entire pattern of results. Since the relations between the memory variables and the theoretical processes underlying them are not necessarily linear, we also analyzed the results of all of the experiments reported in this article using scaled scores. Following Salthouse's 1991 suggestion, we expressed performance in the DA condition in terms of units of the population variability rather than in the original units of measurement. The reference distribution used was the FA condition, which is an appropriate control condition when the standard deviations in this group are similar to those in the DA conditions, as was the case here (see Naveh-Benjamin \& Craik, 1998, for relevant discussion). To obtain standardized scores, we calculated for each participant a score for each of the DA conditions, reflecting performance scaled in standard deviation units of the performance in the FA condition. Results for this experiment, as well as for the following ones, using scaled scores, showed exactly the same patterns as those obtained for the absolute scores.

Secondary task performance. Mean auditory CRT in the baseline condition was $701 \mathrm{msec}$, whereas mean CRT under the DA condition was $1,488 \mathrm{msec}$ (see Table 1). A $t$ test showed this difference to be statistically significant $[t(29)=6.53, p<.01]$.

\section{Discussion}

The corrected hit measure (hits minus false alarms) indicates several patterns. Most important, as indicated by the absence of significant interaction of attention condition and test, participants under DA at encoding did not show differentially poorer performance in recognition of associative information than those under FA. Apparently, DA at encoding affects component processing (either word or nonword) to the same degree as it affects the processing of associations among the components. These results do not support an associative deficit hypothesis as the locus of the effects of DA at encoding on memory performance; DA interrupted component memory to the same degree as it interrupted memory for the associations between the components.

Note that the lack of interaction cannot be attributed to a lack of statistical power of the test. First, the trends obtained do not show any differential effects of DA on the component and the association tests. In fact, the effects of DA were somewhat smaller for the associative than for the component test. Second, the effects of DA were significant in all three tests even though these effects are based on a smaller number of degrees of freedom than the interaction effect.

\section{Experiment 2}

One goal of this experiment was to extend the results of Experiment 1 to other types of associations. To this end,
Table 1

Means and Standard Deviations of Choice Reaction Time in the Baseline and the Divided-Attention (DA) Conditions of the Different Experiments (in Milliseconds)

\begin{tabular}{lccccc}
\hline & \multicolumn{2}{c}{ Baseline } & & \multicolumn{2}{c}{ DA Encoding } \\
\cline { 2 - 3 } \cline { 5 - 6 } Condition & $M$ & $S D$ & & $M$ & $S D$ \\
\hline Experiment 1a & 701 & 407 & & 1,488 & 667 \\
Experiment 2 & & & & \\
$\quad$ Study words & 495 & 97 & & 551 & 121 \\
$\quad$ Study pairs & 495 & 97 & & 558 & 109 \\
Experiment 3a & 962 & 283 & & 1,354 & 331 \\
Experiment 5b & & & & \\
$\quad$ Unrelated pairs & 487 & 76 & & 590 & 121 \\
$\quad$ Related pairs & 487 & 76 & 576 & 110 \\
\hline aAuditory task. bVisual task. & & &
\end{tabular}

the associated pairs in Experiment 2 included words only. A second goal of this experiment was to explore whether an associative deficit under DA at encoding conditions is mediated by the intention to learn the associative information. Information can be encoded and stored incidentally when participants do not encode it in preparation for a given test, or it can be encoded intentionally when participants expect to be tested on this information. Therefore, the second goal of this experiment was to assess whether memory for associations encoded under DA conditions is deficient under both incidental and intentional learning of the associations. Experiment 1 results do not support an associative deficit hypothesis as the locus of the effects of DA at encoding on memory performance under intentional learning of the associations. It is still possible, however, that DA at encoding may differentially affect the registration of associative information when participants concentrate on the encoding of the components and not their associations.

In the present experiment, we again assessed the degree to which DA at encoding differentially affects memory for associative and item information. The type of association used in this experiment was the episodic relations established between two unrelated words that appeared together (inter-unit associations). Word pairs were presented to participants for study under instructions either to learn the items separately or to learn the pairs for an upcoming memory test. As in Experiment 1, item memory was tested by a recognition test in which half of the items had been studied (targets) and half had not (distractors). Associative memory was tested by presenting participants with target items only, either as intact pairs (items presented together at study) or as recombined pairs (items presented in different pairs at study), and asking them to recognize the intact pairs. In addition, study instructions were manipulated so that half of the participants expected an item memory test and half expected an associative memory test. An associative deficit hypothesis predicts an interaction of DA and test, where the differences between FA and DA conditions at encoding are expected to be the greatest in the word-word association test. The words were presented auditorily and participants learned the information 
either alone (FA) or simultaneously with a secondary visual four-CRT task (DA).

\section{Method}

Participants. Participants were 36 undergraduate students at BenGurion University who participated in the experiment as part of their course requirements.

Design. Three independent variables were used: (1) attention (FA vs. DA at encoding; within subjects), (2) test (item vs. pairs; within subjects), and (3) study instructions (study items vs. study pairs; between subjects).

Materials. The materials were similar to those used in Experiment 1 , except that word pairs were used. In the study phase, 56 word pairs were presented auditorily. The first 4 pairs served as practice. Of the next 52 pairs, 4 ( 2 at the beginning and 2 at the end) were used as buffers. The remaining 48 word pairs served as the experimental stimuli. Words belonging to different pairs were not related to each other in any apparent way, either. Three random versions of word pairings were created and two random orders were used for each of these pairings to create six versions. Three participants in each of the study instructions conditions were run under each version.

Procedure. The procedure employed was the same as that used in Experiment 1, with the following changes. Half of the participants were told to study the words in each pair individually in preparation for an upcoming item recognition test, the nature of which was explained (study item instructions). The other half were told to study the word pairs in preparation for an upcoming pair recognition test, whose nature was also explained (study pairs instructions). Altogether, each participant was exposed to one list with half of the items on it presented under FA and the other half presented under DA. The order of the attention conditions was counterbalanced so that, for half of the participants, the first half of the list ( 24 pairs) was presented under FA, and for the other half, the first half of the list was presented under DA.

The secondary task was a visual version of the continuous CRT task. It involved a visual display on a computer screen and a manual response on a computer keyboard (see Craik et al., 1996; NavehBenjamin et al., 1998). The display consisted of four boxes arranged horizontally. An asterisk appeared at random in one of the boxes and the participants' task was to press the corresponding key on the keyboard. Immediately after the participant's correct response, the asterisk moved to one of the other boxes at random.

The study phase involved the presentation of 56 pairs at a rate of 1 pair every $6 \mathrm{sec}$. After an interpolated activity, the two memory tests, one for items and one for their associations, were administered to all participants. The order of the tests was counterbalanced across all participants in each combination of attention and study instructions, and each word or word pair appeared in only one of the tests. The participants also performed the secondary CRT task alone, half before the experimental list and half after it.

1. Item recognition test. The item recognition test was similar to the one employed in Experiment 1 but with 16 targets (half of which were studied under FA and half under DA) and 16 distractors, where half of the targets were the words that had appeared first in a given pair at the study phase and half were words that had appeared second in a given pair. No two words from the same pair were used as targets in this test.

2. Associative recognition test. The associative recognition test was similar to the one used in Experiment 1, with 16 intact (targets) and 16 rearranged (distractor) pairs, half of which were studied under FA and the other half under DA.

\section{Results}

Memory performance. Figure 2 presents results for proportion of hits minus proportion of false alarms in the different conditions. A 2 (attention) $\times 2$ (study instructions) $\times 2$ (test) mixed factorial ANOVA was performed on this measure. There was a significant effect of attention $\left[F(1,34)=22.65, M S_{\mathrm{e}}=.03\right]$, where performance under FA (.58) was better than under DA (.44). The effect of test was also significant $\left[F(1,34)=19.62, M S_{\mathrm{e}}=.046\right]$, where participants' performance on the item test (.59) was better than on the associative test (.43). The effect of study instructions was not significant $\left[F(1,34)=1.23, M S_{\mathrm{e}}=\right.$ .18]. More importantly for the evaluation of an associative deficit hypothesis, the interaction of attention and test was not significant $\left[F(1,34)=0.18, M S_{\mathrm{e}}=.02\right]$, indicating similarly detrimental effects of DA on the item test (.67 and .51, for FA and DA conditions, respectively) and on the associative test (.50 and .37, for FA and DA conditions, respectively). Likewise, no other interactions were significant (all $F_{\mathrm{S}}<1.00$ ). Finally, performance in the DA condition under each combination of study instructions and test was significantly better than chance level (0).

Secondary task performance. Means of the visual CRT task in the baseline and the DA conditions can be seen in Table 1. A two-way ANOVA with attention condition (DA vs. baseline) and instructions (study words vs. study pairs) indicated only a significant effect of attention $\left[F(1,34)=41.47, M S_{\mathrm{e}}=1913.20\right]$, where mean CRT under DA (554 $\mathrm{msec})$ was greater than that in the baseline condition (495 msec).

\section{Discussion}

The results of this experiment are quite clear. Despite the powerful effects of DA on both item and associative recognition, DA at encoding does not result in differentially poorer performance in the recognition of associative information than of item information, when compared with FA. Such a pattern is obtained with both absolute and scaled scores. The absence of both a significant interaction effect of DA and instructions and a significant triple interaction indicates that whatever is disrupted under DA conditions is not related to whether participants pay attention to the components as opposed to their association to each other. Interestingly, paying attention to either the components or to their association to each other does not affect the amount of attentional resources required; secondary task costs, which are a measure of the attentional resources required for encoding, were not affected by encoding instructions. Results of another group of participants, tested under DA at retrieval using the same procedures as those described for the other groups, showed that secondary task performance at retrieval was similar for item $(598 \mathrm{msec})$ and associative $(602 \mathrm{msec})$ recognition tests $[t(35)=0.52$, n.s.]. Such results indicate that execution of these two tests requires the same amount of attentional resources.

To summarize, the results of this experiment, like those reported in Experiment 1, indicate that DA does not differentially disrupt the storage of item versus associative information. 

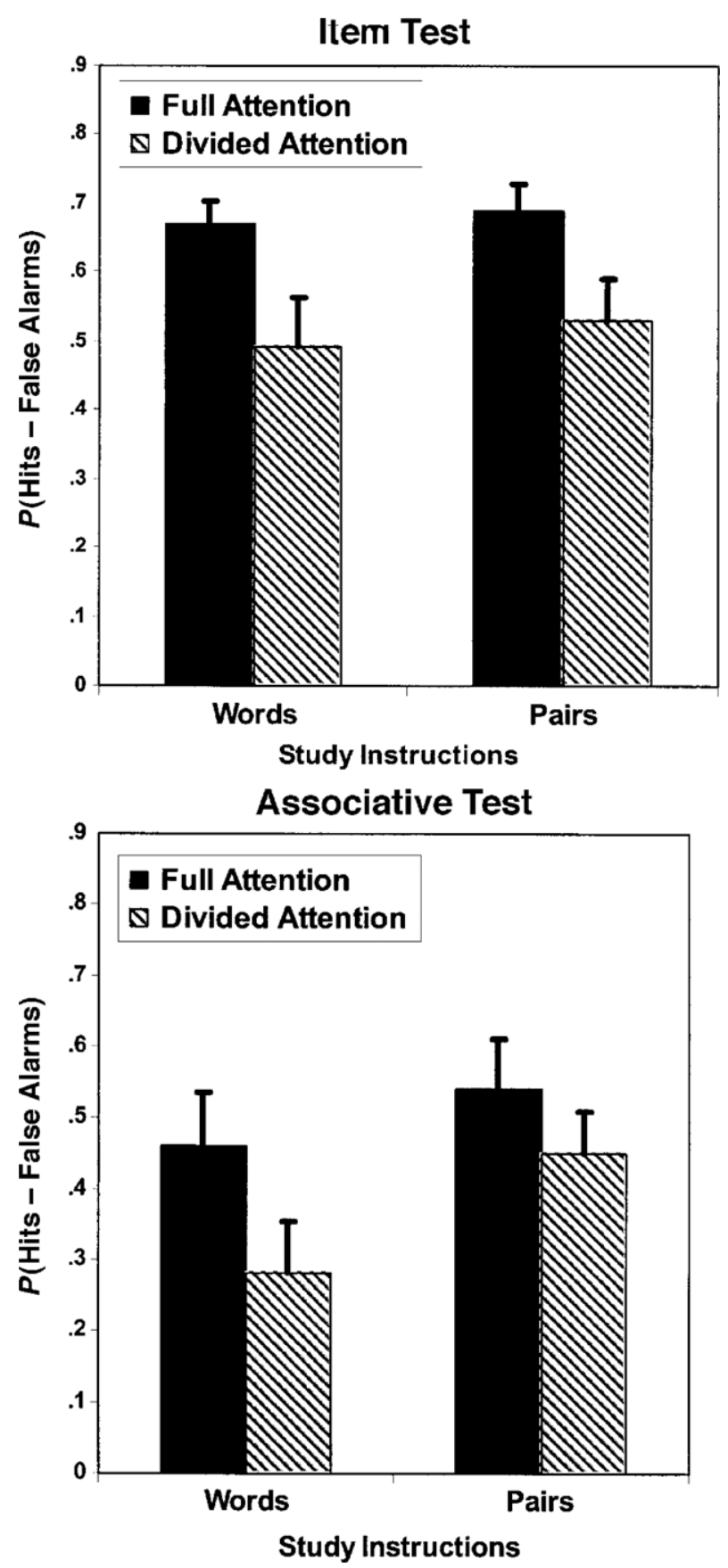

Figure 2. Experiment 2: Proportion hits minus proportion false alarms $(+S E s)$ in the item (words) and the associative (word + word) recognition tests for each attention condition in the different study instruction conditions.

\section{Experiment 3}

Associations are required not only for relating single units (inter-item connections), as discussed and demonstrated in Experiments 1 and 2, but also for connecting together different attributes within a unit. To allow us to evaluate the effects of DA on memory for single attributes and their associations, we devised three memory tasks, each of which is supposed to test a different facet of the information. We used the form (font) in which words were presented as the perceptual-contextual attribute. Words were presented for study in 1 of 18 fonts. We compared FA and DA participants' memory for the words, for the fonts in which the words were presented, and for the relations (conjunctions) between specific words and specific fonts.

For this purpose, at the test phase, participants received the following: (1) In the pure recognition test on the words, some of the original words (targets) appeared with new words (distractors). All of the words at test appeared in a neutral font (which had not been presented at study). (2) In the pure recognition test on the fonts, the original fonts (targets) were mixed with other new fonts (distractors) and were presented without the words (using XXXX) for a recognition test. (3) In the recognition test on the conjunctions/associations of words and fonts, only original (target) words and fonts were presented. In half of the cases, the word was presented in the original font (intact events), and in the other half of the cases, the word was presented in a font different from the one in which it had appeared at study, but one that had appeared with another word at study (recombined events). Such a test requires participants to possess information about the relations between the words and the fonts, and is similar in nature to the recognition test for inter-item associations employed in Experiments 1 and 2.

As in the first two experiments, an associative deficit hypothesis predicts an interaction of attention condition and test, where the differences between FA and DA conditions at encoding are expected to be the greatest in the word-font association test.

\section{Method}

Participants. Participants were 48 undergraduate students at Ben-Gurion University who participated in the experiment as part of their course requirements.

Design. Two independent variables were used: attention (FA, DA; between subjects) and test (words, fonts, words + fonts; within subjects).

Materials. The materials were similar to those used in Experiment 1 , except that 44 words were used. In the study phase, 44 words were presented on index cards, with the first 4 words serving as practice and another 4 as primacy and recency buffers. The remaining 36 words served as the experimental words. For the word memory test, an additional 24 distractor words were chosen. The words had the same characteristic s as those used in Experiments 1 and 2. Twenty-eight experimental fonts were chosen. Eighteen of these fonts were randomly chosen and used as targets, and 10 were used as distractors for the font test. For the study phase, two random versions of word-font pairings ( 36 words, each two appearing in a given font) were created, and three random orders of presentations were used for each of these pairings to create six versions. All test stimuli appeared on index cards.

Procedure. The procedure was similar to the one used in Experiment 1. Participants in each of the attention conditions were told to study the word-font pairs in preparation for tests on the words, fonts, and their combinations. The nature of the tests was explained and demonstrated to them. Presentation rate at study was one word every $6 \mathrm{sec}$. After the practice phase, the experimental phase was run, as in Experiment 1, with 40 experimental items. The secondary task used was the auditory three-CRT task used in Experiment 1. After 
an interpolated activity similar to that used in Experiments 1 and 2, three memory tests were administered to all participants. The order of the tests was counterbalanced across all participants in each of the attention conditions. The number of items in each test was determined in such a way that each word, font, or word-font combination could appear in only one of the tests without repetition. This was done to avoid across-tests item reexposure effects. Participants under the DA condition also performed the secondary CRT task alone, half of them before the experimental trial and half after it.

1. Word recognition test. This test was the same as the one used in Experiments 1 and 2 except that participants saw 36 words, one at a time. Twelve of these were targets and 24 were distractors that were mixed randomly. The use of a one-third proportion of targets was designed to avoid ceiling effects. All of the words appeared in a neutral font - $\mathrm{a}$ font that had not appeared either in the study phase or in the test phase of the font recognition test. Participants were told that 12 of the 36 to-be-presented words had appeared in the study phase and that they should indicate them on the response page provided.

2. Font recognition test. In this test, 10 original fonts that had appeared in the study phase were mixed randomly with 10 distractor fonts and presented one at a time. To avoid any effects of the previously presented words, all fonts appeared using XXXX combinations. Participants were told that 10 of the 20 to-be-presented fonts had appeared at study and that they should indicate which ones they were on the response sheet with which they had been provided.

3. Word-font combination test. In this test, 16 pairs of word-font combinations, all of which had appeared at study, were presented one at a time. To avoid carryover effects from the font test (and vice versa), the words used were those that had appeared in the study phase in one of the eight fonts not used in the font test. Likewise, the fonts used in this test had not appeared in the font test. Eight of the pairs were intact from the study phase; that is, they were word-font pairs that had appeared together in the study phase. The other 8 pairs were rearranged ones; they were composed of words and fonts taken from different study word-font combinations. Participants were told that all of the words and fonts had appeared in the study phase, and were instructed to circle the 8 combinations of the 16 to-be-presented combinations that had appeared as such (intact) in the study phase.

\section{Results}

Memory performance. Figure 3 presents results for proportion of hits minus proportion of false alarm rates. A 2 (attention) $\times 3$ (test) mixed factorial ANOVA was performed on this measure. There was a significant effect of attention $\left[F(1,46)=50.59, M S_{\mathrm{e}}=.049\right]$, where the FA participants (.47) performed better than the DA ones (.22). The effect of test was also significant $[F(2,92)=40.37$, $\left.M S_{\mathrm{e}}=.021\right]$, where participants' performance on the word test (.49) was significantly better than performance on either the font test (.23) or the word-font combination test $(.31)[F(1,46)=35.67$ and 21.76 , respectively $]$. In addition, performance on the word-font association test was better than on the font test $[F(1,46)=6.39]$.

More importantly for the present discussion, the interaction of attention and test was not significant $[F(2,92)=$ $\left.0.15 ; M S_{\mathrm{e}}=.021\right]$; DA participants performed equally poorly in comparison with the FA ones in the word + font combination test as in the separate word test and the font test. Finally, performance of DA participants in each of the tests (.36 in the word test, .11 in the font test, and .18 in the word + font combination test) was significantly better than chance level $(0)$.

Secondary task performance. Mean CRT in the auditory baseline condition was $962 \mathrm{msec}$; mean CRT under the DA condition was $1,354 \mathrm{msec}$ (see Table 1). A $t$ test showed this difference to be statistically significant $[t(23)=5.04, p<.01]$.

\section{Discussion}

The results of this experiment are compatible with those obtained in Experiments 1 and 2. Despite the strong effect of DA on both the components (words and fonts) and their

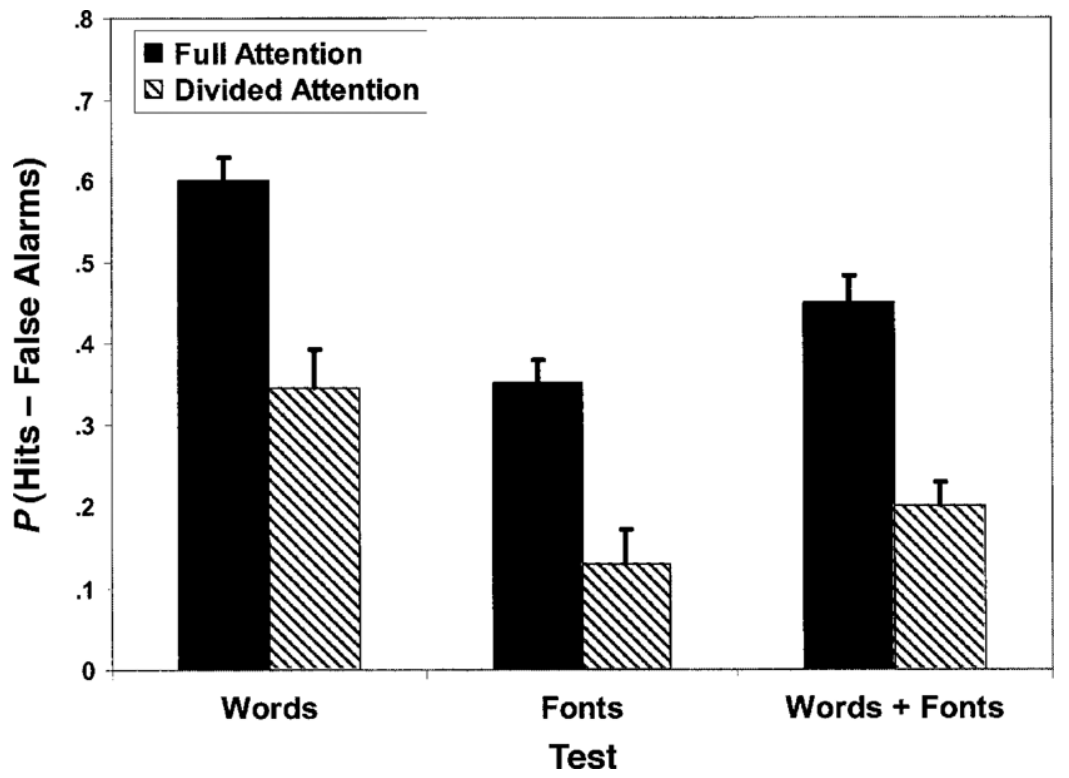

Figure 3. Experiment 3: Proportion hits minus proportion false alarms $(+S E s)$ in the word, font, and word + font recognition tests for each attention condition. 
links to each other, DA at encoding, compared with FA, does not result in a differentially poorer performance in recognition of associative information than item information. This result is evident whether absolute or scaled scores are used. Evidently, DA does not disrupt differentially the storage of component and associative information when this association involves perceptual-contextual attributes.

Overall, these results indicate a similar deficit as a result of encoding the information under DA conditions in a task requiring the association of contextual elements, compared with tasks requiring memory for each of the contextual elements separately. Such results, which extend those reported for inter-unit associations in Experiments 1 and 2, are not consistent with an associative deficit hypothesis as the locus of the DA effects.

\section{Experiment 4}

In this experiment, we compared the effects of DA at encoding on different memory retrieval tasks. Two hypotheses are contrasted: one that attributes the memory deficiency under DA at encoding to a generally poorer encoding of the information, and the other, an associative deficit hypothesis, which attributes this deficit to a selective impairment of the associative mechanism. According to the first hypothesis, the overall poorer encoding under DA conditions will result in a weaker memory trace. This will lead performance under DA at encoding, relative to FA at encoding, to be depressed to the same degree in all of the retrieval tasks. Another version of this hypothesis would predict that this weaker trace under DA at encoding could be helped the most at retrieval when supporting conditions exist such as those present when a recognition test is employed, which could help induce the appropriate mental operations necessary for retrieval (environmental support view, see Craik, 1983, 1986). In a cued recall test, however-and even more so in a free recall test, where participants do not have much support at retrieval and where they have to initiate the mental operations (search, etc.) necessary for recall performance-poor encoding under the DA condition will significantly affect memory performance. Such a view would predict large DA effects at encoding when a free recall task is used, smaller ones when cued recall is used, and still smaller ones when a recognition task is employed, where the poorer encoding under DA can be compensated for by the supporting conditions at retrieval.

In contrast, the associative deficit hypothesis tested here makes different predictions: A cued recall task, which involves the direct encoding and retrieval of specific associations among items, is predicted to be the most sensitive to DA at encoding. This is because participants under DA conditions will encounter problems in creating and retrieving associations between unrelated pairs of items. Free recall is predicted to be somewhat less sensitive to the effects of DA at encoding because it involves other operations not directly related to the retrieval of specific associations (e.g., initiation of a memory search and gener- ation of cues; Atkinson \& Shiffrin, 1968). Finally, item recognition, which is the least dependent on associative information, will be the least DA sensitive. A major difference between the general poor encoding hypothesis and the associative deficit one is in their predictions regarding the effects of DA at encoding on free recall and cued recall performance; whereas the former predicts either no differential effects of DA on the two tasks, or larger effects in free recall, the latter predicts the opposite: larger DA effects in cued recall (see Naveh-Benjamin, 2000). In Experiment 4, we tested these contrasting predictions.

To summarize, in the following experiment we compared the effects of DA at encoding on memory performance in three tasks: free recall, cued recall, and recognition. Participants received six lists of unrelated word pairs to study under either FA or DA, and after each list they performed one of the three memory tasks. Learning was intentional and the nature of the tests was known in advance.

\section{Method}

Participants. Participants were 24 undergraduate students at Ben-Gurion University who participated in the experiment as part of their course requirements.

Design. Two independent variables were used: attention (FA vs. DA at encoding) and type of task (free recall, cued recall, and recognition), both within subjects.

Materials. The study phase for each of the tasks included presentation of 16 pairs of words on index cards. Of the 16 pairs, 4 ( 2 at the beginning and 2 at the end) were used as buffers. The remaining 12 pairs served as the experimental stimuli. For the word recognition test, 24 additional distractor words were chosen. All words were high-frequency two- and three-syllable Hebrew nouns taken from Balgur (1968). The two words in each pair were semantically unrelated to each other . There were no semantic relations between words of different pairs or between words in the different lists. For the study phase, six lists with the above characteristics, one for each task, were created, and completely counterbalanced across the different tasks. Two random orders of presentation at study were used for each of these lists. The secondary task used was the continuous auditory three-CRT task employed in Experiments 1 and 3.

Procedure. Participants, who were run individually, saw six lists of word pairs, one for each combination of attention condition and memory task. For each task, the list of 16 pairs was presented sequentially at study at a rate of one pair every $5 \mathrm{sec}$.

All lists were run under intentional learning instructions. In all lists, participants were told to try to learn the pairs but to pay special attention to the second word in each pair (the target word). They were also told to pay attention to the first cue word because it could help them memorize and retrieve the target word. Participants were told before the beginning of the experimental lists about the three possible memory tasks to be performed, but they were not told in advance which list each was associated with, or that each of the memory tasks would necessarily be employed the same number of times during the experiment. This assured an identical study phase in all tasks in terms of participants' expectations. Responses to questionnaires administered after the experiment indicated no differences under the two attention conditions in terms of the anticipation of the specific upcoming tests.

For the free recall task, participants were told that at the test phase they would have to memorize and write down as many of the targets as possible. For the cued recall task, they were told that at the test phase the first cue word of each pair would be provided and that their task would be to produce its paired word. For the recognition task, 
they were told that at the test phase they would be given the 12 targets plus 24 distractors and that their task would be to circle the 12 targets out of the 36 candidate words.

After a practice phase, for each task, participants were presented with the 16 experimental pairs (including 2 primacy and 2 recency buffer pairs), one at a time without pause. Three of the lists were studied under FA and the other three under DA. After the end of the study phase, participants had to count backward by threes for $60 \mathrm{sec}$ as an interpolated activity. Then the three memory tasks listed below were administered to all participants, one for each list. The order of the tasks across the six lists was counterbalanced across all participants in each combination of attention and test type using a Latin square design. Four participants were assigned to each combination. Finally, participants also performed the secondary auditory threeCRT task alone after the second and the fourth lists for $80 \mathrm{sec}$ each.

1. Free recall task. In this task, participants were asked to write down on the response sheet as many of the target words (the second word in each pair) as possible.

2. Cued recall task. In this task, participants saw each of the cue words in each pair (presented in a random order) and were instructed to write down on the response sheet the target word that appeared with each cue in the study phase.

3. Recognition task. In this task, participants saw 36 words, one at a time. Twelve of these were the targets from the study phase and 24 were distractors that were mixed randomly. The distractors were 24 words with the same characteristics as the target words, but that did not appear in the study phase. Participants were told that 12 of the 36 to-be-presented words had appeared in the study phase as targets and that they should indicate them on a numbered page that was given to them. For all tests, participants had as much time as they needed to provide a response for each test item.

\section{Results}

Memory performance. To compare performance on the three memory tasks directly, we used the proportion of correct targets in the free recall test, the proportion of correct targets in the cued recall test, and the proportion of hits minus the proportion of false alarms in the recognition test (see Craik \& McDowd, 1987, for a similar procedure). Because there were only a few false alarms in the free recall and cued recall tasks, this equated the three tasks in terms of the chance level, which was 0 . These measures were computed for each participant and then averaged over each attention condition.

Figure 4 presents the results for the two attention conditions. A 2 (attention) $\times 3$ (memory task) ANOVA was performed on the memory scores. The effect of DA was significant $\left[F(1,23)=96.78, M S_{\mathrm{e}}=.005\right]$, where performance under FA (.47) was better than under DA (.36). The effect of memory task was also significant $[F(2,46)=$ $\left.54.36, M S_{\mathrm{e}}=.049\right]$. Follow-up contrasts showed that performance in the recognition test (.67) was significantly better than in the cued recall test $(.38)[F(1,23)=31.53]$, which, in turn, was significantly better than performance in the free recall test $(.20)[F(1,23)=10.63]$.

More relevant to our hypotheses, the effect of the interaction of attention condition and memory task was not significant $\left[F(2,46)=1.21, M S_{\mathrm{e}}=.007\right]$. Note that the pattern of those means contradicts the prediction made by the associative deficit hypothesis; the differences between participants under FA and DA were the largest in the free recall test (.14), smaller in the cued recall test (.11), and the smallest in the recognition test (.09) (Figure 4). Performance under DA in all memory tasks was significantly better than chance level (0). In addition, the same results were obtained for scaled scores.

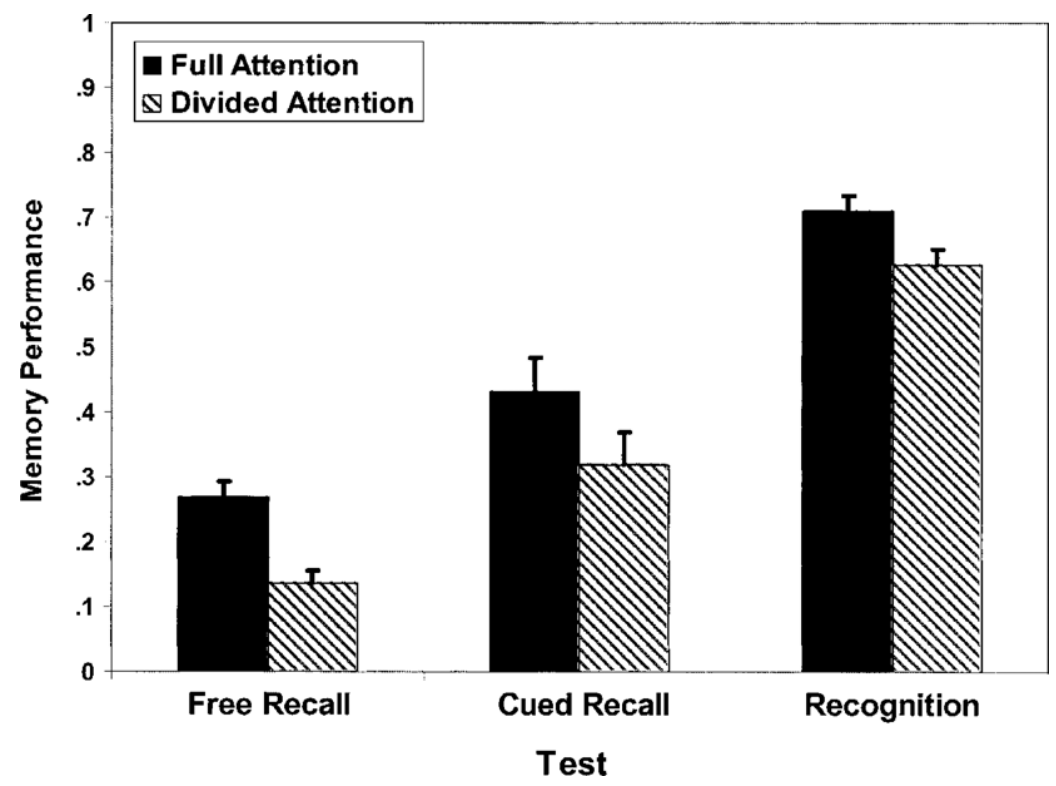

Figure 4. Experiment 4: Proportion of hit rates $(+S E s)$ in the free recall and cued recall tests and proportion hit minus false alarm rates in the recognition test for each attention condition. 
Secondary task performance. Due to equipment failure, results for the secondary task used in this experiment could not be analyzed.

\section{Discussion}

The results of this experiment indicate that participants under DA at encoding are at a general disadvantage when trying to remember unrelated pairs. In particular, it seems that DA at encoding affects performance on the different memory tests to the same degree. Such results, coupled with the trend showing the largest difference between the FA and the DA conditions to be in the free recall task, with a decreasing effect in the cued recall, and an even smaller effect in the recognition tasks, are inconsistent with the associative deficit hypothesis. In particular, according to the associative deficit hypothesis, the cued recall task of unrelated pairs (which involves the direct encoding and retrieval of associations) should prove the most sensitive to the effects of DA at encoding. This, however, was not the case; in fact, the effects of DA were greater for free than for cued recall. Such a pattern, obtained for both absolute and scaled scores, is more consistent with the general "poorer encoding under DA" hypothesis, which predicts, as discussed earlier, either no differences or smaller effects of DA with the addition of external cues (in the cued recall relative to the free recall task).

\section{Experiment 5}

In this experiment, we tested another prediction of the associative deficit hypothesis regarding the underlying mechanism that is disrupted under DA at encoding. Specifically, we contrasted a case where unrelated pairs are employed and new temporal-spatial episodic relations have to be created (as in Experiment 4) with a situation in which the creation and retrieval of these associations can be supported by preexisting semantic associations (e.g., when semantically related pairs are used). In the latter case, where much less episodic distinctiveness is necessary and previous knowledge can support the creation of associations, we would expect much smaller differences, if any, between conditions where participants encode the information under FA or DA. In particular, according to an associative deficit hypothesis, participants under DA should benefit more than those under FA when semantically related rather than unrelated pairs are used in the cued recall task. This is so because the use of preexisting associations will be the most beneficial in encoding associative information under DA. Participants under FA at encoding might also benefit from the semantically related pairs, but to a lesser degree because, according to an associative deficit hypothesis, they encode associative information quite well, even when this information is not supported by preexisting associations.

\section{Method}

Participants. Participants were 38 undergraduate students at Ben-Gurion University who participated in the experiment as part of their course requirements.
Design. Two independent variables were used: attention (FA vs. DA at encoding) and type of pairs (related vs. unrelated semantically), both within subjects.

Materials. The study phase was similar to the one employed in Experiment 4 and included the auditory presentation of 16 pairs of words. The two words in each pair of the unrelated condition were semantically unrelated to each other. The two words in each pair in the related condition were semantically related, using Hebrew norms (Henik \& Kaplan, 1988). In both conditions, there were no semantic relations between words of different pairs or between words in the different lists. For the study phase, four lists with the characteristics mentioned above, two for each pair type, were created. The secondary task was the continuous visual four-CRT task employed in Experiment 2.

Procedure. Participants listened to four lists of word pairs, two for each pair type, one under FA and one under DA at encoding. The order of the attention and type of pairs conditions was counterbalanced using a Latin square design that resulted in 9 or 10 participants run in each of the four combinations. For each task, the list of 16 pairs was presented sequentially at study at a rate of one item every $5 \mathrm{sec}$.

All lists were run under intentional learning instructions to learn the pairs but to pay special attention to the second word in each pair (the target word). Participants were also told to pay attention to the first cue word because it could help them memorize and retrieve the target word. At the beginning of the experimental lists, participants were told about the cued recall task that would follow each list. They were informed that at the test phase, the first cue word of each pair would be provided and their task would be to produce its paired word. Participants were not told in advance whether the pairs were related or unrelated, but they would have noticed that during the practice phase.

After the practice phase, for each list, participants were presented with the 16 experimental pairs (including 2 primacy and 2 recency buffer pairs), one at a time without pause. At the end of the study phase for each list, participants had to count backward by threes for $60 \mathrm{sec}$ as an interpolated activity. Then the cued recall task was administered to all participants. Participants performed the secondary task alone twice, once after the first list and once after the third list.

\section{Results}

Memory performance. We computed for each participant the proportion correct of targets in the cued recall test for each of the lists. Figure 5 presents the means of proportions correct for participants under FA and DA conditions for the different pair types. A 2 (attention) $\times 2$ (pair type) ANOVA performed on the memory scores showed the effect of DA to be significant $[F(1,37)=$ $\left.186.74, M S_{\mathrm{e}}=.008\right]$, where participants under FA (.50) performed better than those under DA (.33). The effect of type of pairs was also significant $[F(1,37)=122.34$, $\left.M S_{\mathrm{e}}=.008\right]$, where performance for related pairs (.52) was better than for unrelated pairs (.31).

More importantly for our hypotheses, the interaction of attention and pair type was not significant $[F(1,37)=$ $\left.1.09, M S_{\mathrm{e}}=.005\right]$, indicating equal memory improvement in both the attention conditions in the related versus the unrelated pairs.

Secondary task performance. Mean visual CRT task in the baseline condition was $487 \mathrm{msec}$; mean CRT task under DA was $583 \mathrm{msec}$ (Table 1). A $t$ test showed this difference to be statistically significant $[t(37)=6.02, p<.01]$. In addition, mean CRT task performance in the unrelated- 
pairs condition $(590 \mathrm{msec})$ was slower than in the relatedpairs condition $(576 \mathrm{msec})[t(37)=2.30, p<.05]$.

\section{Discussion}

The results of this experiment indicate that participants under DA at encoding do not show a differential disadvantage in memory for unrelated pairs. In particular, their deficit for these pairs under DA and under FA was the same as when related pairs were used. Participants under DA clearly take advantage of the related pairs and they do so to the same degree under FA. Such results do not support an associative deficit hypothesis as the locus of the effects of DA at encoding. If associative processes were particularly interrupted under DA at encoding, as an associative deficit hypothesis presumes, we would have expected this to prove especially detrimental to the encoding of unrelated pairs, because they should be harder to associate. The results using both absolute and scaled scores, however, indicated similar interrupting effects of DA for related pairs, in line with the suggestion that associative processes are not differentially interrupted under DA at encoding.

\section{GENERAL DISCUSSION}

The results of the five experiments are not consistent with an associative deficit hypothesis, which attributes at least part of deficient explicit episodic memory performance under DA to decreased ability to encode associations among units of information or attributes within events. The results of the first three experiments, which employed a variety of episodic components and episodic associations, show that DA affected to the same degree memory for the components (measured by a component recogni- tion test) and memory for their associations to each other (measured by an associative recognition test). In Experiment 4 , the demand for associative processing was manipulated at retrieval by having participants engage either in a free recall, a cued recall, or a recognition test, after studying a list of word pairs under FA or DA. Results in this experiment indicated a similar decline in performance in all three memory tasks under DA at encoding condition relative to FA participants. Finally, in Experiment 5, semantically related and unrelated word pairs were studied under either DA or FA. Results indicated similar improvement in memory performance in related relative to unrelated pairs, in both attention conditions.

Although we reported measures of hits minus false alarms for the first three experiments (which used recognition measures), separate measures of hits and false alarms in these experiments showed similar patterns. The reported results do not seem to depend on the scale used; the same patterns emerged regardless of whether we used absolute or scaled scores. In addition, the lack of interaction effect cannot be attributed to a lack of statistical power of the test; first, the trends obtained in all the five experiments do not show any differential effects of DA on the component and the association tests. In fact, in most of the experiments, the effects of DA were somewhat weaker for the associative test than for the component test. Second, the effects of DA were significant in all experiments even though, at least in three of the experiments (1,3, and 4), they are based on a smaller number of degrees of freedom than the interaction effect.

The lack of interaction between attention and test type cannot be attributed to the presentation rates used for each event or to the general robustness of associative informa-

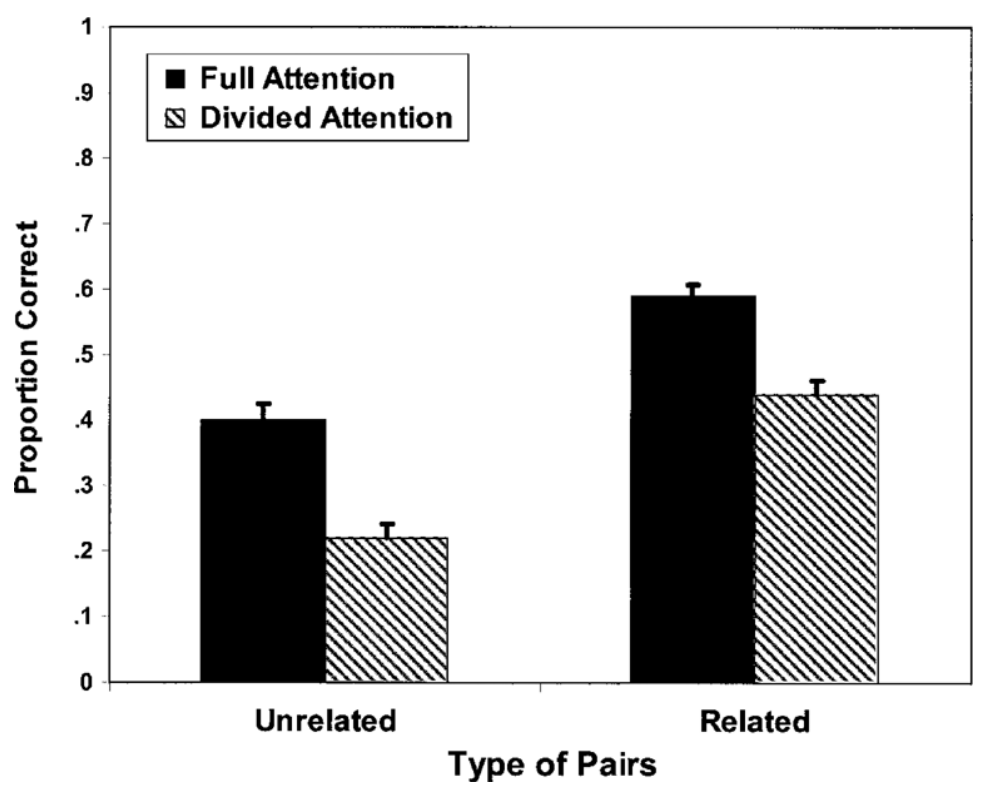

Figure 5. Experiment 5: Proportion correct $(+S E$ s) for each attention condition in the unrelated- and related-pairs condition. 
tion, either. Data collected in our laboratory show that the effects of DA at encoding are similar for item and associative information even when presentation rate is as short as $3 \mathrm{sec}$ per event. In addition, several variables seem to differentially affect associative information, including type of instructions (Hockley \& Christi, 1996), word frequency (Clark, 1992), and age (Naveh-Benjamin, 2000; see discussion below).

The memory performance results indicate similar patterns despite the use of different secondary tasks in the different experiments (continuous visual or auditory CRT tasks). In addition, performance on the secondary task showed similar patterns in all of the experiments, with participants' performance being poorer under DA than in the baseline condition. Such results validate the different secondary tasks employed here by showing that the encoding of the relevant materials diverted attentional resources from the secondary task.

Taken as a whole, the results of the experiments suggest that a deficit in the processing of relations/associations between single units (an episode's components) probably does not play an important role in explaining the poor memory performance of participants who encoded the information under DA conditions. The components can be either two separate units (e.g., words) or more integrated units (a word and its context).

\section{Alternative Mechanisms Involved in the Effects of DA at Encoding on Memory Performance}

As mentioned at the outset, encoding processes have been shown in previous research (Craik et al., 1996; NavehBenjamin et al., 1998; Naveh-Benjamin, Craik, Gavrilescu, \& Anderson, 2000; Naveh-Benjamin, Craik, Perretta, \& Tonev, 2000) to be consciously controlled and attention demanding. There are several information processing stages where the detrimental effects of DA might take place (see Naveh-Benjamin, 2002). One is during the initial registration/perception of the information. Another is during the encoding of the event's components. Others are when the components are bound together, or during the elaboration of the whole episode. The final one might be during the consolidation of the episode. The results of the present study suggest that the effects of DA do not interrupt the binding stage but are either upstream or downstream of it.

Results of recent studies may shed some light on the locus of the effects of DA at encoding. For example, we have recently shown (Naveh-Benjamin, 2002) that DA at encoding affects memory performance similarly regardless of whether the original encoding was performed with the intention to learn or not. Because participants under incidental instructions presumably did not use any encoding strategies (including elaboration), such a pattern of results is consistent with the view that the effects of DA are upstream at an early stage of processing before elaborativestrategic processing takes place, possibly related to the initial registration of the information.
Other studies, however, are consistent with the position that the effects of DA are downstream. For example, recently (Naveh-Benjamin, 2002), we conducted a study where participants were instructed to process the items either at a shallow level (pay attention and make a judgment on the font in which each word appears) or at a deeper level (pay attention to the words and provide a pleasantness rating for each). Results indicated that the effects of DA at encoding were much smaller in the latter condition, indicating that the employment of deep level, elaborative processing may provide immunity to the effects of DA on later memory performance. These elaborations must have been done after the initial encoding was completed; this may imply that DA affects the elaboration of the episode.

It seems, then, that the effects of DA at encoding can interrupt processing either early on, when it is more automatic, or later, when it is more controlled. These effects, however, do not seem to influence the processes involved in associating together the different components of the episodes, as the experiments reported in this article have shown.

There is a least one study in the literature that indicates a somewhat different pattern from the one reported here on the effect of DA at encoding on item and associative information. Reinitz, Morrissey, and Demb (1994) have reported that when participants are presented with faces under FA or DA conditions, performance on a memory test that requires information about conjunctions of facial features was affected by DA to a greater degree than a memory test that required information about specific features. However, their study differs from the ones discussed here in several respects. First, faces are integral-holistic stimuli that may be processed differently from stimuli composed of separate components (see Tanaka \& Sengco, 1997). Recent results (Cooper \& Wojan, 2000) suggest that face identification involves a coordinate shape representation in which the precise locations of visual primitives are specified. This is different from the categorically coded relations that characterize the stimuli used in the present experiments. Second, in their experiment, Reinitz et al. used new distractor faces containing several new features that made the feature (item) recognition test easier than the associative test. Nevertheless, these results indicate that further studies, employing different types of stimuli, both feature-like and holistic, should be conducted to investigate the effects of DA on encoding processes.

\section{The Effects of DA and of Age on Item and Associative Memory}

The results of the present studies are also relevant to questions regarding age-related changes in episodic memory. Craik and his collaborators (e.g., Craik, 1982, 1983, 1986; Craik \& Byrd, 1982) provided one of the most influential approaches to explain age-related changes in memory, claiming that an age-related reduction in attentional or processing resources may underlie the older person's episodic memory deficiency. Moreover, they connected these age-related changes to those occurring in 
younger adults who are operating under conditions of reduced attentional capacity (e.g., DA). The suggestion is that effortful cognitive operations, such as elaboration at encoding and reconstructive operations at retrieval, require substantial attentional resources and that elderly people have fewer of these resources available. According to this suggestion, younger adults under DA presumably invest some of their limited attentional resources in performing the secondary task; operating with reduced attentional resources devoted to the memory task, they would therefore show patterns of memory performance similar to those of older adults. Several studies have supported this common-mechanism hypothesis (e.g., Craik \& Byrd, 1982; Craik \& McDowd, 1987; Craik \& Simon, 1980; Hashtroudi, Johnson, \& Chrosniak, 1990; Hess, Donley, \& Vandermaas, 1989; Rabinowitz et al., 1982), although there are a few studies that are not consistent with this view (e.g., Craik \& Kester, 2000).

Recently, using a procedure similar to the one employed in the present research, Naveh-Benjamin (2000), following Chalfonte and Johnson (1996), provided empirical support to the associative deficit of older adults. In particular, Naveh-Benjamin (2000) conducted four experiments that used the same methodologies employed in the experiments reported in the present manuscript and showed that older adults have a larger differential decline in memory for associations relative to their memory of the components of the episodes. These different patterns in older adults (reported by Naveh-Benjamin, 2000) and in younger adults under DA (reported in this manuscript) are not in line with the suggestion that reduced attentional resources might be a common mechanism underlying the effects of age and the effects of DA in young adults on episodic memory, and place certain constraints on such an explanation. Although both age and DA are negatively associated with episodic memory performance, the loci of their effects seem to be somewhat different. Whereas aging seems to especially disrupt the associative mechanism, reduced attention at encoding in younger adults does not, and is, instead, related to a general decrease in memory performance (see also Naveh-Benjamin, Hussain, Guez, \& Bar-On, 2003).

\section{Summary}

In summary, the experiments reported here, in which participants studied information under either FA or DA and then were tested on their memory for both the episodes' components and the associations between them, indicate clear patterns. Younger adults under DA do not show a differential deficit in memory for associative information; that is, their memory for the components is reduced to the same degree as their memory for the associations among the components. These results, together with previous ones reported in the literature, show that despite robust and replicable empirical support for the detrimental effects of DA at encoding on memory performance, the mechanism that is interrupted by DA during encoding remains unclear. In particular, the present study suggests that the cause of the DA effect at encoding lies somewhere other than in the associative processes that are engaged. One possibility is that DA during encoding does not interrupt any particular process but affects multiple types of processing, resulting in an overall degraded memory trace.

\section{REFERENCES}

Anderson, J. R., \& Bower, G. H. (1973). Human associative memory. Washington, DC: V. H. Winston \& Sons.

Anderson, N. D., Iidaka, T., Cabeza, R., Kapur, S., McIntosh, A. R. \& CRAIK, F. I. M. (2000). The effects of divided attention on encodingand retrieval-related brain activity: A PET study of younger and older adults. Journal of Cognitive Neuroscience, 12, 775-792.

Atkinson, R. C., \& Shiffrin, R. M. (1968). Human memory: A proposed system and its control processes. In K. W. Spence \& J. T. Spence (Eds.), The psychology of learning and motivation: Advances in research and theory (Vol. 2, pp. 89-105). New York: Academic Press.

Baddeley, A. D., Lewis, V., Eldridge, M., \& Thomson, N. (1984). Attention and retrieval from long-term memory. Journal of Experimental Psychology: General, 13, 518-540.

BAlgur, R. (1968). List of basic words for school. Tel Aviv: Otsar Hamoreh.

Chalfonte, B. L., \& Johnson, M. K. (1996). Feature memory and binding in young and older adults. Memory \& Cognition, 24, 403-416.

Clark, S. E. (1992). Word frequency effects in associative and item recognition. Memory \& Cognition, 20, 231-243.

Cooper, E. E., \& Wojan, T. J. (2000). Differences in the coding of spatial relations in face identification and basic-level object recognition. Journal of Experimental Psychology: Learning, Memory, \& Cognition, 26, 470-488.

Craik, F. I. M. (1982). Selective changes in encoding as a function of reduced processing capacity. In F. Klix, J. Hoffman, \& E. Van der Meer (Eds.), Cognitive research in psychology (pp. 152-161). Berlin: DVW.

CrAik, F. I. M. (1983). On the transfer of information from temporary to permanent memory. Philosophical Transactions of the Royal Society of London: Series B, 302, 341-359.

CrAIK, F. I. M. (1986). A functional account of age differences in memory. In F. Klix \& H. Hagendorf (Eds.), Human memory and cognitive capabilities, mechanisms, and performance (pp. 409-422). Amsterdam: Elsevier, North-Holland.

Craik, F. I. M., \& Byrd, M. (1982). Aging and cognitive deficits: The role of attentional resources. In F. I. M. Craik and S. E. Trehub (Eds.), Aging and cognitive processes (pp. 191-211). New York: Plenum.

Craik, F. I. M., Govoni, R., Naveh-Benjamin, M., \& Anderson, N. D. (1996). The effects of divided attention on encoding and retrieval processes in human memory. Journal of Experimental Psychology: General, 125, 159-180.

Craik, F. I. M., \& Kester, J. D. (2000). Divided attention and memory: Impairment of processing or consolidation? In E. Tulving (Ed.), Memory, consciousness, and brain: The Tallinn conference (pp. 38-51). Philadelphia: Psychology Press.

Craik, F. I. M., \& McDowd, J. M. (1987). Age differences in recall and recognition. Journal of Experimental Psychology: Learning, Memory, \& Cognition, 13, 474-479.

Craik, F. I. M., \& Simon, E. (1980). Age differences in memory: The roles of attention and depth of processing. In L. W. Poon, J. L. Fozard, L. S. Cermak, D. Arenberg, \& L. W. Thomson (Eds.), New directions in memory and aging (pp. 95-112). Hillsdale, NJ: Erlbaum.

Dosher, B. A. (1988). Retrieval dynamics of item and associative information. Unpublished manuscript.

Fletcher, P. C., Frith, C. D., Grasby, P. M., Shallice, T., FrackOWIAK, R. S., \& DULAN, R. J. (1995). Brain systems for encoding and retrieval of auditory-verbal memory: An in vivo study in humans. Brain, 118, 401-416.

Gillund, G., \& Shiffrin, R. M. (1984). A retrieval model for both recognition and recall. Psychological Review, 91, 1-67.

Gronlund, S. D., \& Ratcliff, R. (1989). Time course of item and associative information: Implications for global memory models. Journal of Experimental Psychology: Learning, Memory, \& Cognition, 15, 846-858. 
Hashtroudi, S., Johnson, M. K., \& Chrosniak, L. D. (1990). Aging and qualitative characteristics of memories for perceived and imagined complex events. Psychology \& Aging, 5, 119-126.

Henik, A., \& Kaplan, L. (1988). Categories content: Findings regarding Hebrew categories and comparison to US findings. Psychology, $\mathbf{1}$, 104-112.

Hess, T. M., Donley, J., \& Vandermaas, M. O. (1989). Aging-related changes in the processing and retention of script information. Experimental Aging Research, 15, 89-96.

HockLEY, W. E. (1991). Recognition memory for item and associative information: A comparison of forgetting rates. In W. E. Hockley \& S. Lewandowsky (Eds.), Relating theory and data: Essays on human memory in honor of Bennet B. Murdock (pp. 227-248). Hillsdale, NJ: Erlbaum.

HockLey, W. E. (1992). Item versus associative information: Further comparisons of forgetting rates. Journal of Experimental Psychology: Learning, Memory, \& Cognition, 18, 1321-1330.

HoCKLEY, W. E. (1994). Reflections of the mirror effect for item and associative recognition. Memory \& Cognition, 22, 713-722.

Hockley, W. E., \& Christi, C. (1996). Tests of encoding tradeoffs between item and associative information. Memory \& Cognition, 24, 202-216.

Humphreys, M. S. (1976). Relational information and the context effect in recognition memory. Memory \& Cognition, 4, 221-232.

Johnson, M. K. (1992). MEM: Mechanisms of recollection. Journal of Cognitive Neuroscience, 4, 268-280.

Johnson, M. K., \& Chalfonte, B. L. (1994). Binding complex memories: The role of reactivation and the hippocampus. In D. L. Schacter \& E. Tulving (Eds.), Memory systems 1994 (pp. 311-350). Cambridge, MA: MIT Press.

Kapur, S., Craik, F. I. M., Tulving, E., Wilson, A. A., Houle, S., \& Brown, G. M. (1994). Neuroanatomical correlates of encoding in episodic memory: Levels of processing effect. Proceedings of the National Academy of Sciences, 91, 2008-2011.

Mandler, G. (1979). Organization and repetition: Organizational principles with special reference to role learning. In L.-G. Nilsson (Ed.), Perspectives on memory research: Essays in honor of Uppsala University's 500th anniversary (pp. 293-328). Hillsdale, NJ: Erlbaum.

Murdock, B. B., JR. (1965). Effects of a subsidiary task on short-term memory. British Journal of Psychology, 56, 413-419.

Murdock, B. B., JR. (1982). A theory of storage and retrieval of item and associative information. Psychological Review, 89, 609-626.

MuRDock, B. B. (1993). TODAM2: A model for the storage and retrieval of item, associative, and serial-order information. Psychological Review, 100, 183-203.

Naveh-Benjamin, M. (1987). Coding of spatial location information: An automatic process? Journal of Experimental Psychology: Learning, Memory, \& Cognition, 13, 595-605.

Naveh-Benjamin, M. (1988). Recognition of spatial location information: Another failure to support automaticity. Memory \& Cognition, 16, 437-445.

Naveh-Benjamin, M. (1990). Coding of temporal order information:
An automatic process? Journal of Experimental Psychology: Learning, Memory, \& Cognition, 16, 117-126.

Naveh-Benjamin, M. (2000). Adult-age differences in memory performance: Tests of an associative deficit hypothesis. Journal of Experimental Psychology: Learning, Memory, \& Cognition, 26, 1170-1187.

Naveh-Benjamin, M. (2002). The effects of divided attention on encoding processes: Underlying mechanisms. In M. Naveh-Benjamin, M. Moscovitch, \& H. L. Roediger III (Eds.), Perspectives on human memory and cognitive aging: Essays in honorof Fergus Craik (pp. 193207). Philadelphia: Psychology Press.

Naveh-Benjamin, M., \& Craik, F. I. M. (1998). Presenting and analyzing results in aging research. Experimental Aging Research, 24, 83-98.

Naveh-Benjamin, M., Craik, F. I. M, Gavrilescu, D., \& Anderson, N. D. (2000). Asymmetry between encoding and retrieval processes: Evidence from a divided attention paradigm and a calibration analysis. Memory \& Cognition, 28, 965-976.

Naveh-Benjamin, M., Craik, F. I. M., Guez, J., \& Dori, H. (1998). Effects of divided attention on encoding and retrieval processes in human memory: Further support for an asymmetry. Journal of Experimental Psychology: Learning, Memory, \& Cognition, 24, 1091-1104.

Naveh-Benjamin, M., Craik, F. I. M., Perretta, J. G., \& Tonev, S. T. (2000). The effects of divided attention on encoding and retrieval processes: The resiliency of retrieval processes. Quarterly Journal of Experimental Psychology, 53A, 609-625.

Naveh-Benjamin, M., \& Guez, J. (2000). Effects of divided attention on encoding and retrieval processes: Assessment of attentional costs and a componential analysis. Journal of Experimental Psychology: Learning, Memory, \& Cognition, 26, 1461-1482.

Naveh-Benjamin, M., Hussain, Z, Guez, J., \& Bar-On, M. (2003). Adult-age differences in memory performance: Further support for an associative deficit hypothesis. Journal of Experimental Psychology: Learning, Memory, \& Cognition, 29, 826-837.

Naveh-Benjamin, M., \& Jonides, J. (1986). On the automaticity of frequency coding: Effects of competing task load, encoding strategy, and intention. Journal of Experimental Psychology: Learning, Memory, \& Cognition, 12, 378-386.

Rabinowitz, J. C., Craik, F. I. M., \& Ackerman, B. P. (1982). A processing resource account of age differences in recall. Canadian Journal of Psychology, 36, 325-344.

Reinitz, M. T., Morrissey, J., \& Demb, J. (1994). Role of attention in face encoding. Journal of Experimental Psychology: Learning, Memory, \& Cognition, 20, 161-168.

SAlthouse, T. A. (1991). Theoretical perspectives on cognitive aging. Hillsdale, NJ: Erlbaum.

TAnaKa, J. W., \& Sengco, J. A. (1997). Features and their configuration in face recognition. Memory \& Cognition, 25, 583-592.

UNDERWOod, B. J. (1969). Attributes of memory. Psychological Review, 76, 559-573.

(Manuscript received November 26, 2002; revision accepted for publication June 2, 2003.) 